\title{
Hidden QIM Watermarking on Compressed Data Using Channel Coding and Lifting
}

\author{
Santi P. Maity, Claude Delpha, Sofiane Braci, and Rémy Boyer \\ Laboratiore des Signaux et Systèmes (L2S), CNRS, Université Paris-Sud XI (UPS), \\ SUPELEC, France \\ \{santiprasad.maity, claude.delpha, \\ sofiane.braci,remy. boyer \}@lss.supelec.fr
}

\begin{abstract}
This paper investigates the scope of application of channel coding on compressed host data for watermarking using dither modulation (DM) based quantization index modulation (QIM). Lifting based wavelet is used to decompose the encoded compressed data in integer coefficients. The relative gain on imperceptibility and robustness performance are reported for direct watermark embedding on entropy decoded host, using repetition code, convolution code, and finally the combined use of channel codes and lifting. Simulation results show that $6.24 \mathrm{~dB}$ $(9.50 \mathrm{~dB})$ improvement in document- to-watermark ratio (DWR) for watermark power at $12.73 \mathrm{~dB}(16.81 \mathrm{~dB})$ and $15 \mathrm{~dB}$ gain in noise power for watermark decoding at bit error rate (BER) of $10^{-2}$ are achieved, respectively over direct watermarking on entropy decoded data.
\end{abstract}

\section{Introduction}

Nowadays watermarking on compressed host data becomes highly demanding as it is rare to encounter any kind of multimedia signal in a raw, uncompressed format. However, watermarking and compression are antagonistic in characteristics and many watermarking methods developed for the uncompressed host data may not be suitable for the compressed data [1]. Least significant bits (LSB) of variable-length codes (VLC) in MPEG stream [2], JPEG data [3], entropy coded stream [4], and inter-block correlation of the selected DCT coefficients for JPEG compressed data [5], [6] are used for watermarking. Wu et al [7] maximizes robustness in the context of joint watermarking and compression (JWC).

This work applies channel coding on compressed host data for watermarking. Channel coded compressed host data is decomposed by discrete wavelet transform (DWT) using lifting to generate lossless integer coefficients. Watermark information is casted using dither modulation (DM) based quantization index modulation (QIM) for ease of implementation. The relative gain on imperceptibility and robustness performance are reported for direct watermark embedding on entropy decoded host, using repetition code, convolution code, and finally the combined use of convolution code and lifting.

The rest of the paper is organized as follows: Section 2 describes the proposed watermarking method on compressed data. Section 3 presents performance evaluation and discussion. Finally, the paper is concluded in Section 4 along with the scope of future work.

S. Chaudhury et al. (Eds.): PReMI 2009, LNCS 5909, pp. 414-419, 2009.

(C) Springer-Verlag Berlin Heidelberg 2009 


\section{Proposed Watermarking Method on Compressed Data}

Fig. 1(a) and Fig. 1(b) show the basic principle and the block diagram representation of the proposed watermarking scheme, respectively.

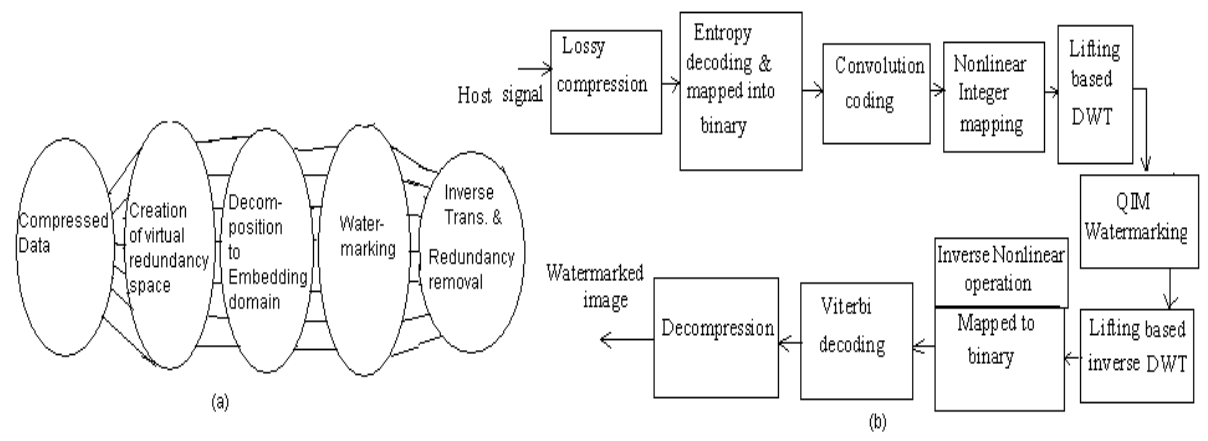

Fig. 1. (a) Basic principle and (b) block diagram representation of the proposed watermarking scheme on compressed data

The lossy compressed host signal is entropy decoded. The non-zero quantized discrete cosine transform (DCT) coefficients are then mapped to the binary data. The binary data so obtained is then encoded using convolution coding to map ' $\mathrm{k}$ ' bits into ' $\mathrm{n}$ ' bits $(n \geq k)$ in order to create a redundancy space in the compressed data and can be made use for watermarking. The convolution code is chosen as it operates on serial data and also uses memory system which in the present case helps to create correlation among the sample coefficients. The channel coded binary data is then converted to integer coefficients through a simple, easily implementable and reversible non-linear mapping like binary-todecimal conversion. The integer coefficients thus obtained undergo lifting based discrete wavelet transform using 5-tap/3-tap filter coefficients [8], however, other lifting based DWT filters can also be used. Lifting-based filtering offers a benefit of very simple filtering operations for which alternately odd sample values of the signal are updated with a weighted sum of even sample values and even samples values are updated with a weighted sum of odd sample values [8] as below.

$$
\begin{gathered}
y(2 n+1)=x_{e s t}(2 n+1)-\left\lfloor\frac{x_{\text {est }}(2 n)+x_{\text {est }}(2 n+2)}{2}\right\rfloor \\
y(2 n)=x_{\text {est }}(2 n)-\left\lfloor\frac{y(2 n-1)+y(2 n+1)+2}{2}\right\rfloor
\end{gathered}
$$

where $x_{\text {est }}$ is the extended input, $y$ is the output signal and $\lfloor a\rfloor$, indicate the largest integer not extending ' $a$ '. Lifting on coded DCT coefficients makes watermarking compatible to both JPEG and JPEG 2000 compression operations. 
A binary message ' $\mathrm{W}$ ' is used as watermark and two dither sequences, with length L, are generated pseudo randomly with step size $(\Delta)$ as follows:

$$
\begin{gathered}
d_{q}(0)=\{\Re(\text { key }) \star \Delta\}-\Delta / 2, \quad 0 \leq q \leq L-1 \\
d_{q}(1)=\left\{\begin{array}{l}
d_{q}(0)+\Delta / 2 \text { if } d_{q}(0)<0 \\
d_{q}(0)-\Delta / 2 \text { if } d_{q}(0) \geq 0
\end{array}\right.
\end{gathered}
$$

where $\Re(k e y)$ is a random number generator. The $q-t h$ watermarked DWT coefficients $S_{q}$ is obtained as follows:

$$
S_{q}=\left\{\begin{array}{l}
Q\left\{X_{q}-d_{q}(0), \Delta\right\}+d_{q}(0) \text { if } W(i, j)=0 \\
Q\left\{X_{q}+d_{q}(1), \Delta\right\}-d_{q}(1) \text { if } W(i, j)=1
\end{array}\right.
$$

where $X_{q}$ is the original channel coded $q$-th DWT coefficients, Q is a uniform quantizer (and dequantizer) with step $\Delta$, and $W(i, j)$ is the $(i, j)$-th pixel of the watermark. Inverse lifting based DWT (IDWT) is then applied on the watermarked coefficients. Inverse non-linear operation maps each integer signal into binary data. The Viterbi decoding is then applied on the binary data to map each 'n'-bits into ' $k$ ' bits. This operation is done for the inverse operation of channel coding i.e. for redundancy removal and not for watermark decoding. The Viterbi decoding is used due to its highly satisfactory bit error performance, high speed of operation, ease of implementation, low cost and fixed decoding time. We call this watermarking as "hidden QIM" as the information embedding process shown in Fig. 1(a) and Fig. 1(b) consist of (i) preprocessing of the compressed host data (using channel coding), (2) QIM embedding, and (3) post processing using channel decoding to form the composite signal [9].

The watermark information can be extracted from the compressed data using the following rule.

$$
\begin{aligned}
A & =\sum_{q=0}^{L-1}\left(\left|Q\left(Y_{q}-d_{q}(0), \Delta\right)+d_{q}(0)-Y_{q}\right|\right) \\
B & =\sum_{q=0}^{L-1}\left(\left|Q\left(Y_{q}+d_{q}(0), \Delta\right)-d_{q}(0)-Y_{q}\right|\right)
\end{aligned}
$$

where $Y_{q}$ is the $q$-th DWT coefficient (possibly noisy due to transmission channel) of the received signal. A watermark bit $\hat{W}(i, j)$ is decoded using the rule:

$$
\hat{W}(i, j)=\left\{\begin{array}{l}
0 \text { if } A<B \\
1 \text { otherwise }
\end{array}\right.
$$

\section{Performance Evaluation and Discussion}

Fig. 2(a) shows $(256 \times 256)$, 8-bits/pixel gray scale watermarked Lena image when binary watermark image of size $(32 \times 32)$ (shown in Fig. 2(b)) is 


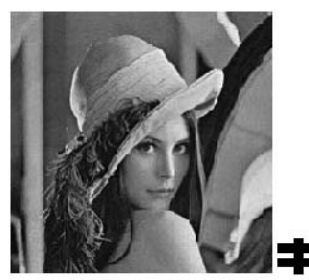

(a)

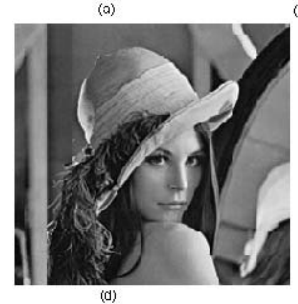

(d)

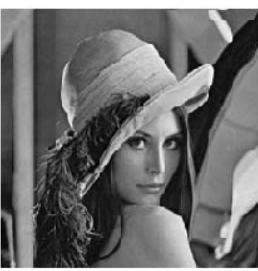

(c)

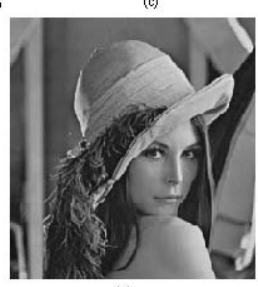

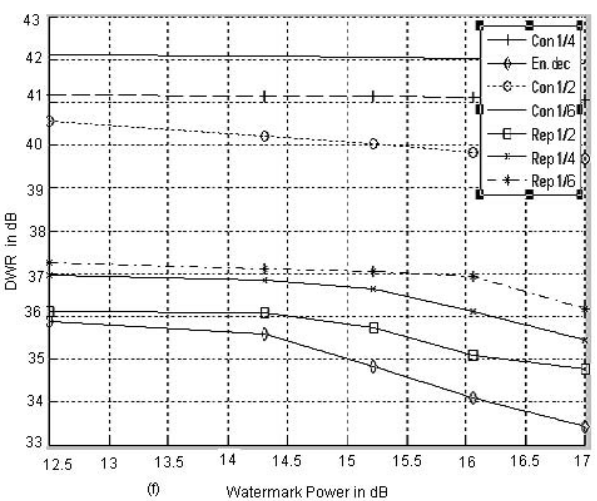

Fig. 2. (a),(c),(d) and (d) Watermarked images after embedding on entropy decoded coefficients, using lifting and convolution codes at rate $1 / 2,1 / 4$ and $1 / 6$, respectively, (b) binary watermark image, and (f) DWR vs watermark power

embedded on entropy decoded DCT coefficients. Although experiment is carried out over large number of images, imperceptibility-robustness performance is reported here only for Lena image due to space limitation. Figs. 2(c)-2(e) show the watermarked images after embedding binary watermark using both lifting and convolution codes at rate $\mathrm{r}=1 / 2,1 / 4$ and $1 / 6$, respectively and at quality factor 60. The numerical values of DWR for the Figs. 2(a), 2(c)-2(e) are $36.89 \mathrm{~dB}$, $41.23 \mathrm{~dB}, 42.67 \mathrm{~dB}$ and $43.12 \mathrm{~dB}$, respectively while the corresponding MSSIM (Mean Structural SIMilarity) index values are 0.9388, 0.9751, 0.9815 and 0.9856, respectively at watermark power $12.73 \mathrm{~dB}$.

Fig. 2(f) and Fig. 3(a) show the graphical representation for DWR with change in watermark power at different code rates. It is quite clear from both the graphs that significant improvement in DWR is achieved due to the use of channel coding compared to the direct embedding of watermark information on the entropy decoded coefficients. The improvement is found to be higher in case of convolution codes compared to the repetition codes. The use of lifting in both cases shows relative improvement in DWR of the order of $\sim 0.5 \mathrm{~dB}$ but benefits in other way. A careful inspection on Fig. 2(f) and Fig. 3(a) show that the use of lifting with channel coding, particularly for convolution coding, maintains high DWR values even with large increase in watermark power leading to a significant improvement in BER performance against AWGN attack. The overall high DWR is achieved due to channel coding which is further augmented through the correlations among the sample coefficients due to the use of lifting and can be well explained by Eq. (1) and Eq.(2).

Fig. 3(b) shows BER performance and can be explained mathematically. The bit error rate $\left(P_{e}\right)$ in watermark detection, for more general case of M-PAM signaling [10], is expressed as 

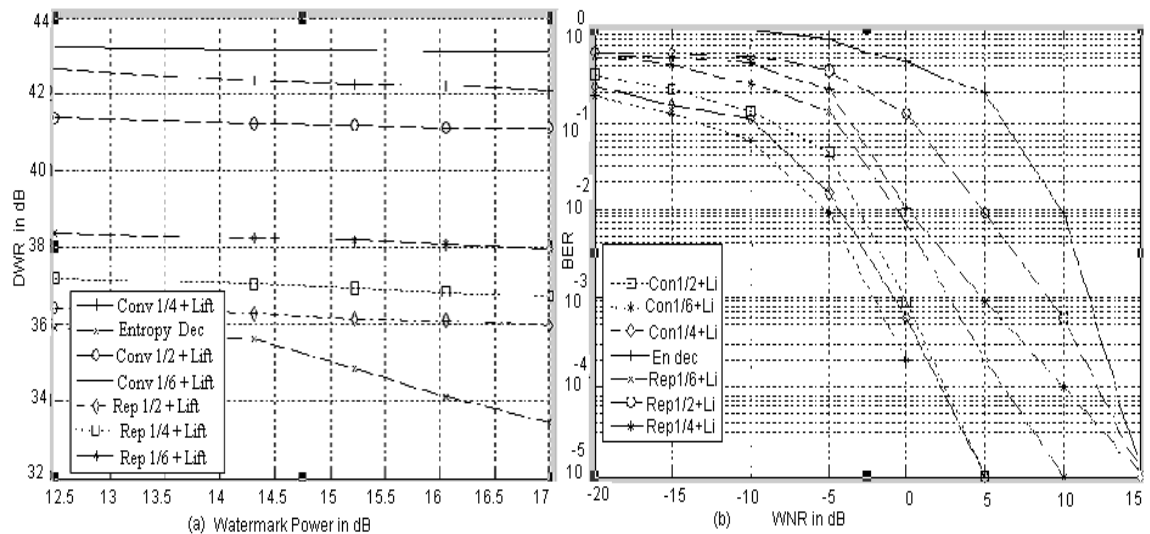

Fig. 3. (a) DWR vs watermark power for direct embedding on entropy decoded data and using both channel coding and lifting, (b)BER performance at different watermarkto-noise (WNR) power in $\mathrm{dB}$

$$
P_{e}=\frac{2(M-1)}{M} \Upsilon\left(\sqrt{\frac{N d_{0}^{2}}{4 \sigma_{x}^{2}}}\right)
$$

where $M$ corresponds to M-PAM (for the present case $\mathrm{M}=2$ ), $N$ is the gain in code rate in terms of the number of cover signal points over which each watermark bit is embedded, $d_{0}^{2}$ indicates the watermark power, $\Upsilon($.$) indicates the$ complementary error function and $\sigma_{x}^{2}$ is the variance of the embedding coefficients. BER performance for watermarking on entropy coded data is poor as in such case $\mathrm{N}=1$ and $\sigma_{x}^{2}$ is high. On the other hand, low $\left(P_{e}\right)$ value for the decoded watermark is achieved due to two-fold advantages, namely large N-values due

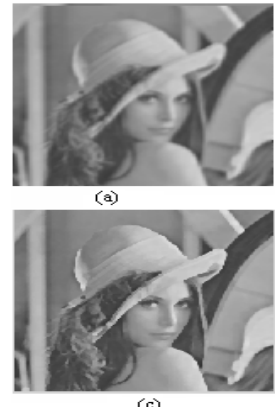

(c)

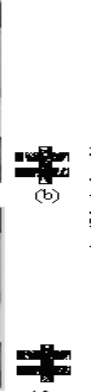

(d)

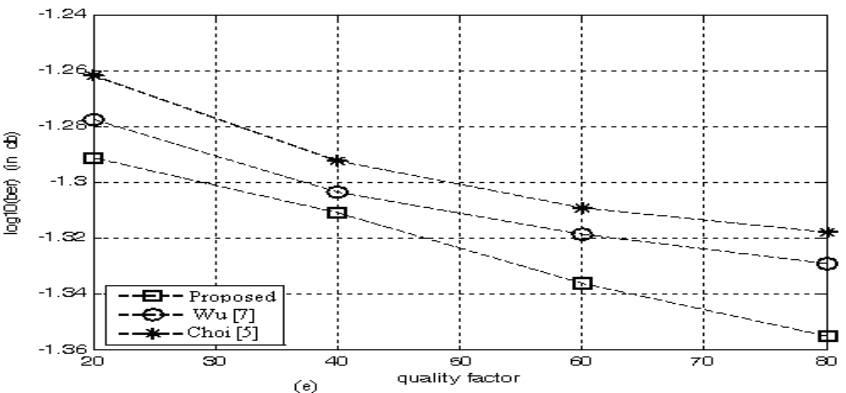

(e)

Fig. 4. (a), (c): Watermarked images after mean and median filtering, respectively, (b)and(d): Extracted watermarks from (a), and (c), respectively, (e) Robustness performance comparison against JPEG compression 
to code rates and low $\sigma_{x}^{2}$ value compared to normal DWT coefficients as integer channel coded data is converted to integer coefficients due to the use of lifting.

Fig. 4(a) and Fig. 4(c) show the watermarked images with DWR $21.41 \mathrm{~dB}$ and $24.03 \mathrm{~dB}$, respectively obtained after mean and median filtering operations using window sizes $(11 \times 11)$, while the extracted watermark images are shown in Fig. 4(b) and 4(d), respectively with BER values 0.0016 and 0.0011 , respectively. Fig. 4(e) shows significantly improved robustness performance for our scheme compared to Choi [5] and Wu [7] method against JPEG compression operation. Simulation results also show similar robustness against JPEG 2000 compressions.

\section{Conclusions and Scope of Future Works}

A novel QIM watermarking is proposed using channel coding and lifting. Channel coding offers improvement both for imperceptibility as well as BER performance while lifting contributes much on BER performance. Present work is going on to implement the proposed algorithm for the compressed audio watermarking application. Future work may be carried out to design capacity optimized hidden watermarking scheme on the compressed data and real-time implementation through VLSI design using FPGA or ASIC.

Acknowledgement. The authors would like to thanks the French National Research program via the ANR project MEDIEVALS for Funding.

\section{References}

1. Wong, P.H., Au, O.C.: A capacity estimation technique for JPEG-to-JPEG image watermarking. IEEE Trans. on Cir. \& Sys. for Video Tech. 13, 747-752 (2003)

2. Langelaar, G.C., Setyawan, I., Lagendijk, R.L.: Watermarking digital image and video data. IEEE Signal Proc. Mag. 17, 20-46 (2000)

3. Fridrich, J., Goljan, M., Chen, Q., Pathak, V.: Lossless data embedding with file size preservation. In: Proc. EI, Security, Steganography, Watermarking Multimedia Contents VI, vol. 5306, pp. 354-365 (2004)

4. Mobasseri, B.J., Berger, R.J.: A foundation for watermarking in compressed domain. IEEE Signal Proc. Lett. 12, 399-402 (2005)

5. Choi, Y., Aizawa, K.: Digital watermarking using interblock correlation. In: Proc. IEEE Int. Conf. Inf. Tech.:Coding and Compr., pp. 133-138 (2000)

6. Luo, W., Heileman, G.L., Pizano, C.E.: Fast and robust watermarking for JPEG files. In: Proc. IEEE 5th Southwest Symp. Image Anal. and Interp., pp. 158-162 (2002)

7. Wu, G., Yang, E.H.: Joint watermarking and compression using scalar quantization for maximizing robustness in the presence of additive gaussian attacks. IEEE Tran. Signal Proc. 53, 834-844 (2005)

8. Adams, M.D., Kossentini, F.: Reversible integer-to-integer wavelet transforms for image compression: performance evaluation and analysis. IEEE Trans. Image Proc. 9, 1010-1024 (2000)

9. Chen, B., Wornell, G.W.: Quantization index modulation: a class of provably good methods for digital watermarking and information embedding. IEEE Trans. on Information Th. 47, 1423-1443 (2001)

10. Voloshynovskiy, S., Pun, T.: Capacity security analysis of data hiding technologies. In: Proc. IEEE Int. Conf. Multimedia and Expo., Laussanne, Switzerland, pp. $477-$ $480(2002)$ 